Published in final edited form as:

Clin Cancer Res. 2019 December 01; 25(23): 7162-7174. doi:10.1158/1078-0432.CCR-19-0302.

\title{
Bioactivation of napabucasin triggers reactive oxygen species- mediated cancer cell death
}

\author{
Fieke E M Froeling 1,2,3,6, Manojit Mosur Swamynathan ${ }^{1,7}$, Astrid Deschênes ${ }^{1}$, lok In \\ Christine Chio ${ }^{1,2,4}$, Erin Brosnan ${ }^{1,2}$, Melissa A Yao ${ }^{1,2,8}$, Priya Alagesan ${ }^{1,2}$, Matthew \\ Lucito $^{1,2}$, Juying $\mathrm{Li}^{5}$, An-Yun Chang ${ }^{5}$, Lloyd C Trotman ${ }^{1}$, Pascal Belleau ${ }^{1}$, Youngkyu \\ Park $^{1,2}$, Harry A Rogoff $5,{ }^{*}$, James D Watson ${ }^{1,{ }^{*}}$, David A Tuveson ${ }^{1,2,{ }^{*}}$ \\ ${ }^{1}$ Cold Spring Harbor Laboratory, Cold Spring Harbor, NY, USA. \\ ${ }^{2}$ Lustgarten Foundation Pancreatic Cancer Research Laboratory, Cold Spring Harbor, NY, USA. \\ ${ }^{3}$ Northwell Cancer Institute, Lake Success, NY, USA \\ ${ }^{4}$ Institute for Cancer Genetics, Columbia University, New York, NY, USA. \\ ${ }^{5}$ Boston Biomedical Inc., Cambridge, MA, USA. \\ ${ }^{6}$ Department of Surgery and Cancer, Imperial College London, London, UK. \\ ${ }^{7}$ Graduate Program in Molecular and Cellular Biology, Stony Brook University, Stony Brook, NY, \\ USA. \\ ${ }^{8}$ Icahn School of Medicine at Mount Sinai, New York, NY, USA.
}

\begin{abstract}
Purpose-Napabucasin (2-acetylfuro-1,4-naphthoquinone or BBI-608) is a small molecule currently being clinically evaluated in various cancer types. It has mostly been recognized for its ability to inhibit STAT3 signaling. However, based on its chemical structure, we hypothesized that napabucasin is a substrate for intracellular oxidoreductases and therefore may exert its anti-cancer effect through redox cycling, resulting in reactive oxygen species (ROS) production and cell death.

Experimental Design-Binding of napabucasin to NAD(P)H:quinone oxidoreductase-1 (NQO1), and other oxidoreductases, was measured. Pancreatic cancer cell lines were treated with napabucasin, and cell survival, ROS generation, DNA damage, transcriptomic changes and alterations in STAT3 activation were assayed in vitro and in vivo. Genetic knock-out or pharmacological inhibition with dicoumarol was used to evaluate the dependency on NQO1.

Results-Napabucasin was found to bind with high affinity to NQO1 and to a lesser degree to cytochrome P450 oxidoreductase (POR). Treatment resulted in marked induction of ROS and DNA damage with an NQO1- and ROS-dependent decrease in STAT3 phosphorylation.

Differential cytotoxic effects were observed, where NQO1-expressing cells generating cytotoxic
\end{abstract}

Corresponding author: David A. Tuveson, Cold Spring Harbor Laboratory, 1 Bungtown Road, Cold Spring Harbor, NY 11724, USA. Phone: 516-367-5246; Fax: 516-367-8353; dtuveson@ @ cshl.edu.

${ }^{*}$ These authors contributed equally.

Conflict of interest: Juying Li, An-Yun Chang, and Harry Rogoff are salaried employees of Boston Biomedical, Inc. James D Watson previously acted as a Consultant to Boston Biomedical, Inc. 
levels of ROS at low napabucasin concentrations were more sensitive. Cells with low or no baseline NQO1 expression also produced ROS in response to napabucasin, albeit to a lesser extent, through the one-electron reductase POR.

Conclusions-Napabucasin is bioactivated by NQO1, and to a lesser degree by POR, resulting in futile redox cycling and ROS generation. The increased ROS levels result in DNA damage and multiple intracellular changes, one of which is a reduction in STAT3 phosphorylation.

\section{Keywords}

napabucasin; NQO1; oxidoreductases; reactive oxygen species; cytotoxicity

\section{INTRODUCTION}

Under physiological conditions, incomplete reduction of oxygen results in the production of reactive oxygen species (ROS), including hydrogen peroxide $\left(\mathrm{H}_{2} \mathrm{O}_{2}\right)$, the superoxide anion $\left(\mathrm{O}_{2}^{-}\right)$and the hydroxyl radical $(\bullet \mathrm{OH})$. To protect molecules from ROS-induced damage, cells orchestrate a complex network of antioxidants to maintain proper cellular function. This reduction-oxidation (redox) balance is tightly controlled by several key transcription factors including nuclear factor erythroid-derived 2-like 2, NFE2L2/NRF2, which regulates the transcription of a number of target genes encoding components of antioxidant systems, glutathione synthesis enzymes, proteasome subunits and heat-shock proteins (1-3).

Disruption of this delicate redox balance has long been known to be associated with multiple diseases, including cancer development and progression (4). Tumors are thought to harbor a unique state of redox regulatory mechanisms to support their pathological survival and proliferation, demonstrating a biphasic response. At low levels, ROS are mutagenic and can promote tumor development by activating signaling pathways that regulate cellular survival, proliferation, differentiation and metabolic adaptation. However, at high levels, ROS become toxic leading to oxidative stress and cell death or senescence $(1,5)$. To compensate for higher levels of intrinsic ROS, cancer cells have evolved adaptive mechanisms that increase their antioxidant capacity. NRF2 upregulation has been observed in multiple tumor types and its expression has been shown to be required for pancreatic and lung cancer development (5-7). Thus, compared to normal cells, cancer cells with increased oxidative stress are likely more vulnerable to damage by further ROS insults, making modulation of tumor redox homeostasis an attractive therapeutic strategy.

The NRF2 target gene NAD(P)H:quinone oxidoreductase 1 (NQOI) is a two-electron oxidoreductase involved in the detoxification of quinones using NADH or NADPH to generate the corresponding hydroquinone derivative (8). Increased expression of NQO1 has been observed in many solid tumors, has been shown to occur early in tumorigenesis and has been linked to multiple carcinogenic processes (9-15). For example, increased NQO1 expression is observed in precursor lesions (pancreatic intraepithelial neoplasia) and further increased expression occurs in invasive pancreatic ductal adenocarcinoma (13-15). The ability of NQO1 to generate hydroquinones, combined with its overexpression in many cancers, has been utilized as a therapeutic strategy and various anti-cancer compounds that are bioactivated by NQO1 have been developed. Hydroquinones can exhibit toxicity through a number of mechanisms, depending on their chemical reactivity. Bioactivation of anti- 
tumor quinones such as mitomycin $\mathrm{C}$ or streptonigrin results in hydroquinone-mediated alkylation of DNA with interstrand crosslinking (16). In contrast, oxidoreduction of naphthoquinones, such as $\beta$-lapachone, results in an unstable hydroquinone that spontaneously reacts with oxygen to regenerate the original compound in a two-step back reaction, depleting $\mathrm{NAD}(\mathrm{P}) \mathrm{H}$ and generating substantial amounts of $\operatorname{ROS}(17,18)$.

Napabucasin, also known as BBI-608, is an orally administered small molecule that is being clinically evaluated for the treatment of a variety of cancers, including pancreatic ductal adenocarcinoma $(19,20)$. It is mostly recognized for its ability to inhibit signal transducer and activator of transcription 3 (STAT3)-mediated gene transcription with activity against bulk tumor cells and cancer stem cells, with inhibition of spherogenesis in vitro and tumor relapse in vivo (21-23). However, the mechanism by which napabucasin mediates these effects is not understood. In this report, we sought to further elucidate its mechanism of action based on the notion that napabucasin is a naphthoquinone (2-acetylfuro-1,4naphthoquinone). We show that napabucasin is a substrate for NQO1, and to a lesser degree for the one-electron reductase Cytochrome $\mathrm{P} 450$ reductase (POR). Bioactivation of napabucasin results in ROS generation, inducing oxidative stress and DNA damage with multiple ROS-induced intracellular events including, but not limited to, a reduction in STAT3 phosphorylation.

\section{METHODS}

\section{Cell lines}

Cell lines were obtained from ATCC, or JCBR (Suit2) or generated from established human organoids as previously described (24) and cultured in DMEM (10-013-CV, Fisher Scientific) or RPMI (10-040-CV, Fisher Scientific) containing 10\% FBS. All cells were cultured for no more than 20 passages and tested negative for mycoplasma using the MycoAlert Mycoplasma Detection Kit (LT07-318, Lonza). Cell line authentication was not performed.

NQO1 knock-out CRISPR clones from MiaPaCa2, AsPc1, and DU145 cell lines were generated as previously described using Lenti_sgRNA_EFS_GFP (LRG) plasmids (Addgene \#65656) $(25,26)$. sgRNAs targeting unique locations at the NQO1 locus were designed, cloned and validated by Sanger sequencing. Non-targeting sgRosa was used as a control. Cas9-expressing cells were infected and sorted for GFP expression on the FACSAria cell sorter (BD). For $N Q O 1$ knock-out in FaDu cells, the parental cell line was transfected with ribonucleoprotein (RNP) complexes composed of sgRNA and Cas9NLS protein using manufacturer's instructions (Thermo Fisher Scientific). In brief, functional sgRNA was generated by annealing tracrRNA and crRNA. A 1:1 ratio of sgRNA and Cas9NLS protein was mixed with LipoCas9 plus reagent and incubated for 5 minutes at room temperature to produce an RNP complex. The RNP complex was then mixed with Lipofectamine CRISPRMAX transfection reagent and added to the parental cell cultures. Following overnight incubation, the culture medium was replenished, and cells were expanded until a sufficient quantity of genomic DNA could be extracted. Successful gene editing was verified by heteroduplex analysis. Potential NQO1 knock-out clones were selected and complete $N Q O 1$ knock-out was verified by Western blot. For expression of 
NQO1 in Panc1 cells, NQO1 was introduced by transfection of cDNA (Origene, RC200620) using XtremeGENE 9 (Roche, 06365787001) according to manufacturer instructions. Functional assays were performed 36 hours post transfection with a CMV-driven GFP expressing plasmid as control. In MDA-MB-231 cells, $N Q O 1$ was introduced using lentiviral transduction followed by blasticidin selection as directed by the manufacturer (GenTarget).

\section{Expression and purification of NQO1}

The coding sequence for human $N Q O 1$ was synthesized and cloned into pET15b (Novagen) using BamHI and NdeI restriction sites (Genewiz), along with an N-terminal hexahistidine affinity tag and thrombin cleavage site (MGSSHHHHHHSSGLVPRGSH).

BL21(DE3)pLysS Escherichia coli (Promega) were transformed with plasmid and grown at $37^{\circ} \mathrm{C}$ in Luria-Bertani medium supplemented with $100 \mu \mathrm{g} / \mathrm{mL}$ ampicillin to an optical density at $600 \mathrm{~nm}$ of 0.8 . Cultures were then chilled to $18^{\circ} \mathrm{C}$, and protein expression was induced overnight with $0.5 \mathrm{mM}$ isopropyl $\beta$-D-1-thiogalactopyranoside. Cells were harvested, and lysate was loaded onto Ni-NTA affinity resin equilibrated in $50 \mathrm{mM}$ HEPES (pH 7) supplemented with $0.15 \mathrm{M}$ sodium chloride. Resin was washed extensively, and protein was eluted with buffer plus $0.25 \mathrm{M}$ imidazole. NQO1 was further purified with a Hiload 16/600 Superdex200 pg column (GE Healthcare); protein purity was judged to be $>95 \%$ by SDS-PAGE. NQO1 was flash frozen for subsequent analysis.

\section{Cell-free assays}

Initial rates of NQO1 substrate digestion $(0.4-25 \mu \mathrm{M})$ were monitored using an assay in which the oxidation of NADPH to NADP+ was quantified at $340 \mathrm{~nm}$ at $30^{\circ} \mathrm{C}$ using Spectramax 5 (Molecular Devices). Reactions of $0.02 \mu \mathrm{M}$ NQO1, $800 \mu \mathrm{M}$ NADPH in 50 $\mathrm{mM}$ potassium phosphate ( $\mathrm{pH} 7.4$ ), and $5 \%$ DMSO with or without $5 \mathrm{mM}$ dicoumarol were initiated by addition of NADPH. Wells were monitored every 3 seconds for 2 minutes to obtain an initial linear signal that was converted to " $\mu \mathrm{M}$ NADPH per minute per $\mu \mathrm{M}$ NQO1" using a standard curve. Michaelis-Menten curves were generated with GraphPad Prism 5. Reactions were performed in triplicate. Similar reactions were carried out with purified NADPH:cytochrome P-450 reductase (POR) (C8113, Sigma), carbonyl reductase 1 (CBR1) (ab85336, Abcam), and thioredoxin (TRX1) (ab51064, Abcam).

\section{Napabucasin dose-response curves}

Cells were plated at $\sim 70 \%$ confluency and increasing concentrations of napabucasin (range $0.01-5 \mu \mathrm{M}$ ) as single agent or combined with the antioxidant N-acetylcysteine (NAC), the ROS scavenger EUK-134 (Sigma) or the NQO1 inhibitor dicoumarol (Selleckchem) were added in triplicate 24 hours after plating and normalized to DMSO. Cell viability was assessed following 6 hours of treatment using CellTiter-Glo® (Promega). Dose-response curves were generated using GraphPad Prism 5.

\section{Measurement of ROS generation}

ROS generation with simultaneous assessment of cell viability or changes in total to oxidized glutathione ratios following napabucasin treatment were determined by the ROS- 
$\mathrm{Glo}^{\mathrm{TM}} \mathrm{H}_{2} \mathrm{O}_{2}$ (Promega) or GSH/GSSG-Glo ${ }^{\mathrm{TM}}$ assay (Promega), respectively, as per manufacturer's instructions. In brief, cells were seeded in 96-well plates the day prior to treatment such that drug treatment was added when cells were 50-80\% confluent. For ROS$\mathrm{Glo}^{\mathrm{TM}} \mathrm{H}_{2} \mathrm{O}_{2}$ assays, culture medium was replaced with $100 \mu \mathrm{L}$ medium containing $25 \mu \mathrm{M}$ $\mathrm{H}_{2} \mathrm{O}_{2}$ substrate plus the desired drug concentration. After incubating for 6 hours at $37^{\circ} \mathrm{C}, 50$ $\mu \mathrm{L}$ of supernatant were transferred to a new 96-well plate containing an equal volume of ROS detection reagent. A total of $50 \mu \mathrm{L}$ CellTiter-Glo® reagent (Promega) was added to the 96-well plate containing the remaining $50 \mu \mathrm{L}$ of culture. For GSH/GSSG-Glo ${ }^{\mathrm{TM}}$ assays, cells were treated for 6 or 24 hours. Following treatment, medium was removed, and cells were washed with Hank's Balanced Salts and lysed with either total or oxidized glutathione reagent. Cell lysis was followed by luciferin generation and detection after which luminescence was read.

Measurements of GSH and GSSG in snap-frozen tumor samples was done by adapting the procedures described by Moore et al (27). Briefly, snap-frozen tissue specimens were lysed, incubated for 45 minutes at room temperature to allow derivatization of GSH to GSH-NEM after which supernatant was collected. For GSH measurements, $5 \mu \mathrm{L}$ of derivatized sample was mixed with $50 \mu \mathrm{L}$ of GSH-NEM standard ([13C2,15N]-glutathione, $200 \mu \mathrm{M})$, vortexed and transferred into autosampler glass vials. Similarly, sample extracts were added to an equal volume of GSSG internal standard solution ([13C4,15N2]-glutathione disulfide) for GSSG measurements. Samples were randomized in order to avoid bias due to machine drift and processed blindly. LC-MS analysis was performed using a Q Exactive HF mass spectrometer coupled to a Vanquish Horizon UHPLC system (Thermo Fisher Scientific). The acquired spectra were analysed using XCalibur Qual Browser and XCalibur Quan Browser software (Thermo Fisher Scientific). Absolute quantification was performed by interpolation of the corresponding standard curve obtained from serial dilutions of commercially available standards run with the same batch of samples.

For ROS measurement by chloromethyl $\mathrm{H}_{2}$ DCFDA, cells were washed with PBS, labeled with $5 \mu \mathrm{M} \mathrm{CM}-\mathrm{H}_{2}$ DCFDA (ThermoFisher) for $30 \mathrm{~min}$ and analyzed by flow cytometry.

\section{In vivo subcutaneous transplantation}

Nude mice were purchased from Charles River Laboratory (stock number 24102242) and $20 \mu \mathrm{l}$ of $5.0 \times 10^{5} \mathrm{MiaPaCa} 2 \mathrm{Rosa} 26$ or MiaPaCa2 NQO1-71 cells mixed within an equal volume of PBS and Matrigel were injected subcutaneously. Tumor-bearing mice with a tumor volume of $150 \mathrm{~mm}^{3}\left(0.5 \mathrm{x}\right.$ length $\mathrm{x}$ width $\left.{ }^{2}\right)$ were enrolled on a randomized basis to start treatment with either napabucasin dissolved in $0.5 \%$ methylcellulose or $0.5 \%$ methylcellulose. Mice were dosed once daily by oral gavage at $200 \mathrm{mg} / \mathrm{kg}$ for 24 days with monitoring of tumor volume every 3 days. All animal procedures were conducted in accordance with the Institutional Animal Care and Use Committee at Cold Spring Harbor Laboratory (CSHL).

\section{Western blot analysis}

Whole cell lysates were prepared at baseline or following 2 hours of drug treatment in a lysis solution of $20 \mathrm{mM}$ HEPES, 300mM NaCl, 5mM EDTA, 10\% Glycerol and 20\% Triton 
$\mathrm{X}-100, \mathrm{pH} 7.5$, supplemented with protease Mini-complete protease inhibitors (11836170001, Roche) and a phosphatase inhibitor cocktail (4906845001, Roche). Standard procedures were followed for Western blotting using the following primary antibodies: Actin (8456, Cell Signaling Technology), STAT3 (9139, Cell Signaling Technology), pSTAT3 (9145, Cell Signaling Technology), pJAK1 (3331, Cell Signaling Technology), JAK1 (MAB42601-SP, R\&D), pJAK2 (3771, Cell Signaling Technology), JAK2 (3230, Cell Signaling Technology), NQO1 (3187, Cell Signaling Technology), POR (ab13513, Abcam), $\beta$-Tubulin (2148, Cell Signaling Technology), Catalase (12980, Cell Signaling Technology) and NRF2 (ab62352, Abcam). Proteins were detected using HRP-conjugated secondary antibodies (Jackson ImmunoResearch Laboratories).

\section{Immunofluorescence}

Cells were fixed with $3.7 \%$ formaldehyde, permeabilized with $0.1 \%$ Triton-X100, blocked with $0.1 \%$ BSA and incubated for 1 hour at room temperature with phospho-histone H2A.X antibody (9718, Cell Signaling Technology) followed by Alexa488 or Alexa647-labelled secondary antibody and DAPI as counterstain. Imaging was performed with a Leica TCS SP8 laser scanning confocal microscope (Boulder Grove Il).

\section{RNA-sequencing and analysis}

Following 2 hours of treatment with $0.5 \mu \mathrm{M}$ napabucasin or DMSO, cells were lysed using TRIzol Reagent (15596-018; Thermo Fisher Scientific) and RNA was extracted with a PureLink RNA mini kit (12183018A; Thermo Fisher Scientific). Libraries were prepared using a KAPA mRNA HyperPrep Kit for Illumina sequencing (Roche, KR1352-v4.17) according to manufacturer's instructions and single-end RNA-sequencing was performed on an Illumina NextSeq500. All RNA-sequencing data are available at the Gene Expression Omnibus (GEO) under the accession number GSE135352.

Differential gene expression analysis was performed using Bioconductor package DESeq2 (28), with a pre-filtering step to remove genes that had no reads or reads only in one sample. Only genes with an adjusted p-value $<0.05$ and a $\log 2$ fold change $>=1$ were retained as significantly differentially expressed. Gene Set Enrichment Analysis (GSEA) (29) was performed to evaluate napabucasin-mediated alterations in the HALLMARK IL6-JAKSTAT3 geneset specifically. Additional functional enrichment analysis was performed by creating protein-protein and Reactome pathway-protein interaction networks using Search Tool for Retrieval of Interacting Genes/Proteins (STRING) version 11.0 (30), stringApp version 1.4.2 (31) and Cytoscape version 3.7.1 (32).

\section{RNA interference}

Synthetic, small-interfering RNA (siRNA) oligos targeting NQO1, NQO2, POR, Ferredoxin Reductase (FDXR), Cytochrome B5 Reductase 1 (CYB5RI), Cytochrome B5 Reductase 3 (CYB5R3), Cytochrome B5 Reductase 4 (CYB5R4), Carbonyl Reductase 1 (CBRI), and Thioredoxin Reductase 1 ( TXNRDI) were obtained from Ambion. Cells were transfected with siRNA using Lipofectamine RNAiMAX (Invitrogen) and assayed at 72 hours posttransfection. 


\section{qPCR analysis}

Samples were lysed with TRIzol Reagent, with homogenization for snap frozen tumor samples, and RNA was extracted with a PureLink RNA mini kit (12183018A; Thermo Fisher Scientific) followed by reverse transcription of $1 \mu \mathrm{g}$ RNA using TaqMan reverse transcription reagents (N808-0234; Applied Biosystems). qPCR was performed using genespecific TaqMan probes (Applied Biosystems) and master mix (4440040; Applied Biosystems). Gene expression was normalized to HPRT or ACTIN. siRNA knock-down was verified by qPCR with RT-qPCR primers (Qiagen) and iTaq universal SYBR green supermix (Bio-Rad) on CFX connect real-time system (Bio-Rad).

\section{RESULTS}

\section{Napabucasin activity and ROS generation}

Given that napabucasin was originally hypothesized to target cancer cells and cancer stem cells by reduction of STAT3 signaling $(20,21)$, we first determined whether these activities were also observed in a panel of pancreatic cancer cell lines. When cells were treated for 6 hours with increasing concentrations of napabucasin, differential cytotoxicity was observed (Fig. 1A). Reductions in the active, phosphorylated form of STAT3, as well as phosphorylated JAK1 and JAK2, were observed, but to a different degree for each cell line (Fig. 1B). Based on its naphthoquinone structure (Supp. Fig. 1A), we hypothesized that napabucasin may function as a ROS generator through NQO1-mediated redox cycling, and that reduced JAK/STAT signaling may be a downstream effect of napabucasin-mediated ROS production. Indeed, treatment with napabucasin increased ROS levels and reduced cell viability (Fig. 1C, D, E, F), which was mitigated by the addition of the antioxidant $\mathrm{N}$ acetylcysteine (NAC) (Fig. 1G). Of note, cells in which relative levels of napabucasininduced ROS were higher (MiaPaCa2 and AsPc1) were found to be more sensitive to napabucasin compared to those with less napabucasin-induced ROS generation (Suit2 and Panc1), although higher concentrations of napabucasin were required for AsPc1 cells (Fig. 1D, E). Nevertheless, generation of ROS, as measured by a change in the ratio of the antioxidant glutathione (GSH) to its oxidized species (GSH disulfide [GSSG]), in response to a fixed, low, dose of napabucasin $(0.5 \mu \mathrm{M})$, correlated with response (Fig. 1F). Similar observations were made in colon and lung cancer cells (Supp. Fig. 1B), with a rescue in cell viability when napabucasin was combined with the ROS scavenger EUK-134 (Supp. Fig. 1C).

\section{Napabucasin is an NQO1 substrate}

To determine whether napabucasin can act as a substrate for NQO1-mediated reduction using NADPH, we assessed NQO1 substrate digestion in a cell-free system in which we quantified the oxidation of NADPH to NADP+ when NQO1 was incubated with either napabucasin or the known NQO1 substrate $\beta$-lapachone as a control $(18,33)$. Napabucasin was shown to directly bind to human NQO1 with high catalytic activity. This effect was blocked by dicoumarol, a specific NQO1 inhibitor that competes with NADH/ NADPH substrate binding (Fig. 2A). Moreover, compared to $\beta$-lapachone, napabucasin had tighter NQO1 binding affinity $\left(\mathrm{K}_{\mathrm{M}}\right)$ and better catalytic efficiency $\left(\mathrm{k}_{\mathrm{cat}} / \mathrm{K}_{\mathrm{M}}\right)($ Fig. $2 \mathrm{~A})$, suggesting that napabucasin is a more potent substrate of NQO1. 
We next evaluated NQO1 expression in a panel of pancreatic cancer cell lines (Fig. 2B) and whether pharmacological inhibition of NQO1 could reverse the napabucasin-mediated effects. Combined treatment of napabucasin and dicoumarol rescued cell viability in MiaPaCa2, AsPc1, and organoid-derived pancreatic cancer cell lines (Fig. 2C, Supp. Fig. 2A). In contrast, combination treatment did not rescue viability in Suit2 or Panc1 cells (Fig. 2C), due to the undetectable levels of NQO1 protein in these cells (Fig. 2B). To determine whether dicoumarol also prevented napabucasin-mediated ROS production, we measured $\mathrm{H}_{2} \mathrm{O}_{2}$ levels and the GSH:GSSG ratio in cells treated with napabucasin and/or dicoumarol. The dicoumarol-mediated rescue in cell viability inversely correlated with changes in the levels of ROS generation: napabucasin-mediated increases in ROS levels were inhibited by dicoumarol in MiaPaCa2, AsPc1, and organoid-derived cell lines, but not in the NQO1deficient Suit2 or Panc1 cell lines (Fig. 2D, E, Supp. Fig. 2B).

To further assess the dependency of napabucasin activity on NQO1, we used CRISPR/Cas9 to knock-out $N Q O 1$ in the pancreatic cancer cell lines MiaPaCa2 and AsPc1 (Fig. 3, Supp. Fig. 3A, B, C), as well as DU145 cells, a metastatic prostate cancer cell line (Supp. Fig. 3D, E) and FaDu cells, a hypopharynx squamous cell carcinoma cell line (Supp. Fig. 3D, F). $N Q O 1$ ablation made cells more resistant to napabucasin (2.5 - 3.5 fold) and to a lesser degree to $\beta$-lapachone (1.5 fold) (Fig. 3A, B and Supp. Fig. 3A). The reduced activity of napabucasin in NQO1-ablated cells was associated with decreased ROS induction, as measured by $\mathrm{H}_{2} \mathrm{O}_{2}$ levels (Fig. 3C) and a shift in the GSH:GSSG ratio (Fig. 3D). Similar observations were made in subcutaneous xenografts, with intra-tumoral ROS generation, as detected by reduced GSH:GSSG ratios, in MiaPaCa2 Rosa26 xenografts treated with napabucasin but not in tumors derived from NQO1 knock-out cells (Supp. Fig. 3B, C). The NQO1-dependency of napabucasin was also observed in DU145 and FaDu cells (Supp. Fig. 3D, E, F). Similarly, ectopic expression of NQO1 in the NQO1-negative Panc1 cells and a NQO1-negative breast cancer cell line MDA-MB-231, sensitized these cells to napabucasin with an associated increase in ROS production (Fig. 3E, F and Supp. Fig. 3G).

Taken together, these results show that napabucasin induces ROS in human tumor cells in an NQO1-dependent manner, and suggest that napabucasin-mediated cytotoxicity may be dependent on both ROS and NQO1 expression.

\section{Napabucasin activity and changes in STAT3 signaling}

Given the role of NQO1 and ROS in napabucasin-mediated cytotoxicity, and the observed decrease in phosphorylation of STAT3 (pSTAT3) upon napabucasin treatment (Fig. 1B), we sought to determine whether NQO1 expression and ROS generation were required to inhibit activation of the STAT3 pathway. In MiaPaCa2 cells, with high baseline pSTAT3, we found that $N Q O 1$ knock-out predominantly restored pSTAT3 expression in napabucasin treated cells, as did the addition of the NQO1 inhibitor dicoumarol (Fig. 3G). However, in AsPc1 cells that have much lower basal levels of pSTAT3 (Fig. 1B), napabucasin treatment did not diminish pSTAT3 levels in an NQO1-dependent manner (Supp. Fig. 3H). Similarly, while there were no changes in pSTAT3 upon treatment of the NQO1-deficient MDA-MB-231 breast cancer cells with napabucasin, the re-introduction of NQO1 to MDA-MB-231 cells was sufficient to restore the ability of napabucasin to diminish pSTAT3 levels (Supp. Fig. 
$3 \mathrm{G})$. Additionally, treatment with $\mathrm{H}_{2} \mathrm{O}_{2}$ was sufficient to reduce pSTAT3 expression in all pancreatic cancer cell lines (Fig. 3H, Supp. Fig. 3I), and pSTAT3 levels were partially restored in MiaPaCa2 cells when napabucasin was combined with NAC (Fig. 3H). These data indicate that napabucasin mediated inhibition of STAT3 activity is a secondary effect from the treatment-induced high levels of ROS, which is, in part, dependent on NQO1 expression.

\section{Napabucasin-induced transcriptomic changes}

Based on the notion that napabucasin induces ROS in an NQO1 dependent manner, resulting in ROS-driven intracellular signaling modifications, we further evaluated the transcriptomic changes following 2 hours of treatment with napabucasin in MiaPaCa2 cells, two NQO1 knock-out clonal lines (NQO1-71 and NQO1-163) and the respective Rosa26 control. In the parental MiaPaCa2 cells a total of 158 genes were differentially expressed, with the majority of genes being upregulated following treatment with napabucasin (Supp. Fig. 4A, Supp. Table 1). Of those 158 genes, 24 showed an NQO1-dependent differential expression, including many genes known to be induced upon cellular stress (Fig. 4A). Surprisingly, there was no significant, NQO1-dependent enrichment of the JAK-STAT signaling pathway, with only three genes from the JAK-STAT geneset significantly enriched in the napabucasin treated parental MiaPaCa2 cells $(H M O X 1, M A P 3 K 8$, SOCS3; FDR corrected $\mathrm{p}=0.02$; Supp. Fig. 4B). Heme oxygenase ( $H M O X 1)$ is a well-known NRF2 target gene, which expression is known to be induced by ROS to protect cells against oxidative damage by catalyzing the breakdown of heme molecules and sequestering the redox-active $\mathrm{Fe}^{2+}(3,34,35)$. HMOX1 expression was strongly induced upon treatment with napabucasin in an NQO1-dependent manner, both in vitro and in vivo, with increased expression of $H M O X 1$, as well as other NRF2 target genes, in the NQO1 positive MiaPaCa2 and AsPC1 cells (Fig. 4A, B, C) or tumors from MiaPaCa2 xenografts (Fig. 4D), but not in the NQO1 knock-out MiaPaCa2 cells or xenografts or the NQO1 negative Suit2 and Panc1 cells Fig. 4 A, C, D).

Additional protein-protein (Supp. Fig. 4C) and pathway-protein interaction network (Fig. 4B) analysis with the differentially expressed genes in the parental MiaPaCa2 cells further highlighted the induction of oxidative stress and DNA damage upon treatment with napabucasin, with upregulation of the stress response genes $A T F 3$ and $A T F 4$, as well as other members of the AP1 transcription complex (FOS, JUN) and early response genes involved in cell cycle arrest in response to DNA damage (CDKN1A, BTG1, BTG2) (Fig. 4B). This ROS-induced stress response upon treatment with napabucasin was seen across cell lines and in the MiaPaCa2 Rosa26, but not in the MiaPaCa2 NQO1 knock-out, xenografts (Fig. 4D, E).

\section{Napabucasin and NADPH:cytochrome P-450 reductase (POR)}

Based on the observation that napabucasin still has an effect in NQO1-deficient cells, with a reduction in cell viability and ROS generation, albeit at a lesser degree compared to NQO1expressing cells, we hypothesized that the antitumor effects of napabucasin may also be conferred via NQO1-independent pathway(s). Indeed, there are several non-NQO1 reductases with the potential to generate ROS from quinones (Supp. Fig. 5A) (36, 37). To this end, we examined the interactions between napabucasin (and $\beta$-lapachone) and a 
number of one-electron reductases: NADPH:cytochrome P-450 reductase (POR), carbonyl reductase 1 (CBR1) and thioredoxin 1 (TRX1). In a cell-free system, both napabucasin and $\beta$-lapachone were shown to be substrates of POR (Fig. 5A). Additional evaluation showed that napabucasin and $\beta$-lapachone have different specificities for the other reductases studied. For example, while both napabucasin and $\beta$-lapachone can be efficiently reduced by NQO1 and POR, $\beta$-lapachone can also be reduced by CBR1, while CBR1 has little activity against napabucasin (Fig. 5B).

To determine whether POR can substitute for NQO1 as the reductase that acts on napabucasin in NQO1-deficient cells, we used RNA interference (siRNA) to deplete various reductases in Panc1 cells, which do not express detectable NQO1 protein but do express POR (Fig. 2B, Supp. Fig. 5B, C). Of the siRNAs screened, siRNA directed against POR inhibited napabucasin-mediated cell death to the greatest extent (Fig. 5C), with an associated reduction in ROS generation (Fig. 5D). Interestingly, knock-down of some oxidoreductases sensitized Panc1 cells to napabucasin, an effect most profoundly observed with knock-down of the NRF2 target gene thioredoxin reductase 1 (TXNRDI) (Fig. 5C). The increased sensitivity to napabucasin seen when TXNRD1 was knocked down was accompanied by elevated ROS production (Fig. 5D). These results highlight the intricate regulation of intracellular oxidative stress and suggest that in the absence of NQO1, napabucasin may be a substrate for POR, which can generate ROS and mediate cell death. Conversely, other cellular reductases (e.g. TXNRD1) may function as antioxidants, inhibiting the cytotoxic activity of napabucasin.

In conclusion, our data indicate that napabucasin is bioactivated by NQO1, with a role for the one-electron reductase POR in cells that do not express NQO1. This, in turn, results in increased ROS generation causing DNA-damage (Fig. 6A, Supp. Fig. 6) and a multitude of intracellular events including a reduction in STAT3 phosphorylation, stabilization of NRF2 (Supp. Fig. 7) with upregulation of NRF2 target genes as well as the activation of other stress-induced genes and protective mechanisms in an attempt to counteract the ROSinduced damage (Fig. 6B). Given the redox difference between cancer cells and normal cells, the high expression of NQO1 in many cancers, including pancreatic cancer, makes disruption of this balance by napabucasin an attractive, tumor-specific approach.

\section{DISCUSSION}

Here, we show that the naphthoquinone napabucasin can be bioactivated by the cellular reductases NQO1 and, to a lesser extent, POR, resulting in the production of ROS and disruption of the cellular redox balance, resulting in DNA-damage induced cell death. While traditionally ROS are considered to be toxic molecules causing indiscriminate damage to proteins, nucleic acids and lipids, it is increasingly recognized that they also play a significant role as secondary messengers in cellular signaling (37). A number of transcription factors contain redox-sensitive cysteine residues at their DNA binding sites, including nuclear factor- $\mathrm{kB}$ (NF- $\mathrm{kB}$ ), HIF-1 and p53. In addition, ROS can either inhibit or activate protein function through altering their phosphorylation status via thiol oxidation of either tyrosine phosphatases or kinases $(1,38,39)$. Similar to previous reports $(21-23)$, we observed a decrease in STAT3 phosphorylation upon treatment with napabucasin in 
pancreatic and breast cancer cells. However, the ability to do so appeared to be dependent on NQO1 and ROS generation. Indeed, STAT3 phosphorylation can be inhibited directly or indirectly by ROS $(40,41)$ but in the absence of a reduction in JAK-STAT signaling in response to napabucasin, the functional importance of the reduction in PSTAT3 expression remains unclear. Instead, the decrease in pSTAT3 is most likely a secondary event in response to increased ROS and may serve as a pharmacodynamic biomarker in which high baseline pSTAT3 expression may also be predictive of response. Consistently, early data has shown improved survival in patients with advanced, pSTAT3-positive colorectal cancer treated with napabucasin compared to placebo (42).

Redox alterations in cancer cells are complex, in which cancer cells have become adapted to higher levels of oxidative stress resulting in malignant transformation, metastasis and drug resistance. Drug-resistant cancer cells may use redox regulatory mechanisms to promote cell survival and tolerate external insults from anti-cancer agents. Therapeutically increasing ROS levels by agents such as napabucasin, may cause cells to lose their "stemness", rendering them drug sensitive (43). Although this currently is an unexplored area, it is evident that ROS generation plays a critical role in the anti-tumor activity of napabucasin. The use of NQO1 as a predictive biomarker for sensitivity to napabucasin, or other quinone anti-cancer drugs, is appealing. However, NQO1 protein levels are not stable and can for example be induced by a host of dietary components or environmental factors (16). In addition, we observed a differential response to napabucasin also within the NQO1 positive cells. In particular, AsPc1 cells required the highest drug concentration to induce ROSmediated cell death with temporal changes in response when cells were treated for a longer period of time (data not shown). Despite of previous reports indicating expression of the antioxidant catalase as important mechanism of resistance to $\beta$-lapachone in NQO1 positive cells $(33,44)$, we did not observe such a correlation with regards the response to napabucasin (Supp. Fig. 7A). Gene and protein expression analysis however showed marked NRF2 pathway activation after only 2 hours of drug exposure (Fig. 4, Supp. Fig. 7B) and ROS-induced upregulation of various cytoprotective mechanisms may play a role in the temporal kinetics of response. Moreover, we observe that cells that do not express NQO1 are still able to generate ROS following napabucasin treatment, although to a lesser degree, through cytochrome P450 oxidoreductase (POR), an oxidoreductase known to be the source of ROS generation resulting in paraquat-induced cell death (36). The precisely coordinated, and dynamic regulation of ROS generation and detoxification is further highlighted by the different effects of napabucasin when expression of various oxidoreductases is reduced by siRNA. In particular, reduction of the antioxidant thioredoxin reductase 1 (TXNRD1) enhanced napabucasin activity and concomitantly increased ROS production. Thus, a more comprehensive "redox-signature" may be better predictive of tumors likely to respond to napabucasin, rather than the expression of a single protein.

The thioredoxin system is an important thiol antioxidant, consisting of thioredoxin (TRX) and thioredoxin reductase, frequently upregulated in cancer (45). To maximally exploit ROS-mediated cell death mechanisms, combining napabucasin with agents that inhibit the thioredoxin pathway, such as sulfasalazine or auranofin $(46,47)$, may further enhance its anti-tumor activity. Many conventional cytotoxic cancer drugs can also directly, or indirectly increase ROS levels in cancer cells and may synergize with napabucasin. Current clinical 
trials are testing this hypothesis. For instance, the combination of napabucasin, gemcitabine and nab-paclitaxel is currently being evaluated as a treatment for metastatic pancreatic cancer (CanStem111P, ) (19). However, as with all anti-cancer therapies, identifying responsive subgroups is paramount in order to significantly improve clinical outcomes. Our study provides important insights regarding the mechanism of action of napabucasin, which will assist further biomarker development and research aimed to identify optimal therapeutic combination approaches with identification of those patients who are most likely to benefit from napabucasin.

\title{
Supplementary Material
}

Refer to Web version on PubMed Central for supplementary material.

\section{ACKNOWLEDGMENTS}

\begin{abstract}
We would like to thank the Cold Spring Harbor Cancer Center Support Grant (CCSG) shared resources: E. Ghiban in the Next Generation Sequencing Core Facility, P. Moody and C. Kanzler in the Flow Cytometry Facility, S. Costa at Mass Spectrometry Core Facility, Q. Gao at Histology Core Facility, and all staff at the Animal Facility. The CCSG is funded by the NIH Cancer Center Support Grant 5P30CA045508. This work was supported by the Lustgarten Foundation, where D.A. Tuveson is a distinguished scholar and Director of the Lustgarten Foundationdesignated Laboratory of Pancreatic Cancer Research. D.A. Tuveson is also supported by the Cold Spring Harbor Laboratory Association and the David Rubinstein Center for Pancreatic Cancer Research at MSKCC, the V Foundation, the Thompson Foundation and the Simons Foundation (552716). In addition, this work was supported by the National Institutes of Health, NIH P30CA045508, P50CA101955, P20CA192996, U10CA180944, U01CA168409, U01CA210240, R33CA206949, R01CA188134 and R01CA190092 to D.A. Tuveson. We are also grateful for support from the Donaldson Charitable Trust for F.E.M. Froeling and The Northwell Health Affiliation for F.E.M Froeling and D.A. Tuveson. Y. Park is supported by the National Institute of Health (R50CA211506). I.I.C. Chio is supported by Pancreatic Cancer Action Network (PG009667 - PANCAN 18-35-CHIO); the V Foundation (PG009685 - VFND V2018-017); and Columbia University Medical Center (Paul Marks Scholar Award). We thank Dr. Lindsey Baker and Dr. Claudia Tonelli for critical review of the manuscript. We also thank Dr. Tom Miller for the original observation that napabucasin is a quinone.
\end{abstract}

\section{REFERENCES}

1. Trachootham D, Lu W, Ogasawara MA, Nilsa RD, Huang P. Redox regulation of cell survival. Antioxidants \& redox signaling. 2008;10(8):1343-74. [PubMed: 18522489]

2. Rojo de la Vega M, Chapman E, Zhang DD. NRF2 and the Hallmarks of Cancer. Cancer Cell. 2018;34(1):21-43. [PubMed: 29731393]

3. Tonelli C, Chio IIC, Tuveson DA. Transcriptional Regulation by Nrf2. Antioxidants \& redox signaling. 2018;29(17):1727-45. [PubMed: 28899199]

4. Cross CE, Halliwell B, Borish ET, Pryor WA, Ames BN, Saul RL, et al. Oxygen radicals and human disease. Ann Intern Med. 1987;107(4):526-45. [PubMed: 3307585]

5. Kong H, Chandel NS. Regulation of redox balance in cancer and T cells. J Biol Chem. 2018;293(20):7499-507. [PubMed: 29282291]

6. Chio IIC, Jafarnejad SM, Ponz-Sarvise M, Park Y, Rivera K, Palm W, et al. NRF2 Promotes Tumor Maintenance by Modulating mRNA Translation in Pancreatic Cancer. Cell. 2016;166(4):963-76. [PubMed: 27477511]

7. DeNicola GM, Karreth FA, Humpton TJ, Gopinathan A, Wei C, Frese K, et al. Oncogene-induced Nrf2 transcription promotes ROS detoxification and tumorigenesis. Nature. 2011;475(7354):106-9. [PubMed: 21734707]

8. Ross D, Siegel D. NAD(P)H:quinone oxidoreductase 1 (NQO1, DT-diaphorase), functions and pharmacogenetics. Methods in enzymology. 2004;382:115-44. [PubMed: 15047100]

9. Bey EA, Bentle MS, Reinicke KE, Dong Y, Yang CR, Girard L, et al. An NQO1- and PARP-1mediated cell death pathway induced in non-small-cell lung cancer cells by beta-lapachone. Proc Natl Acad Sci U S A. 2007;104(28):11832-7. [PubMed: 17609380] 
10. Bentle MS, Reinicke KE, Dong Y, Bey EA, Boothman DA. Nonhomologous end joining is essential for cellular resistance to the novel antitumor agent, beta-lapachone. Cancer Res. 2007;67(14):6936-45. [PubMed: 17638905]

11. Yang Y, Zhang Y, Wu Q, Cui X, Lin Z, Liu S, et al. Clinical implications of high NQO1 expression in breast cancers. Journal of experimental \& clinical cancer research : CR. 2014;33:14. [PubMed: 24499631]

12. Belinsky M, Jaiswal AK. NAD(P)H:quinone oxidoreductase1 (DT-diaphorase) expression in normal and tumor tissues. Cancer Metastasis Rev. 1993;12(2):103-17. [PubMed: 8375015]

13. Chakrabarti G, Silvers MA, Ilcheva M, Liu Y, Moore ZR, Luo X, et al. Tumor-selective use of DNA base excision repair inhibition in pancreatic cancer using the NQO1 bioactivatable drug, beta-lapachone. Sci Rep. 2015;5:17066. [PubMed: 26602448]

14. Silvers MA, Deja S, Singh N, Egnatchik RA, Sudderth J, Luo X, et al. The NQO1 bioactivatable drug, beta-lapachone, alters the redox state of NQO1+ pancreatic cancer cells, causing perturbation in central carbon metabolism. J Biol Chem. 2017;292(44):18203-16. [PubMed: 28916726]

15. Li LS, Bey EA, Dong Y, Meng J, Patra B, Yan J, et al. Modulating endogenous NQO1 levels identifies key regulatory mechanisms of action of beta-lapachone for pancreatic cancer therapy. Clin Cancer Res. 2011;17(2):275-85. [PubMed: 21224367]

16. Siegel D, Yan C, Ross D. NAD(P)H:quinone oxidoreductase 1 (NQO1) in the sensitivity and resistance to antitumor quinones. Biochem Pharmacol. 2012;83(8):1033-40. [PubMed: 22209713]

17. Pink JJ, Planchon SM, Tagliarino C, Varnes ME, Siegel D, Boothman DA. NAD(P)H:Quinone oxidoreductase activity is the principal determinant of beta-lapachone cytotoxicity. J Biol Chem. 2000;275(8):5416-24. [PubMed: 10681517]

18. Reinicke KE, Bey EA, Bentle MS, Pink JJ, Ingalls ST, Hoppel CL, et al. Development of betalapachone prodrugs for therapy against human cancer cells with elevated $\mathrm{NAD}(\mathrm{P}) \mathrm{H}$ :quinone oxidoreductase 1 levels. Clin Cancer Res. 2005;11(8):3055-64. [PubMed: 15837761]

19. Bekaii-Saab TS, Starodub A, El-Rayes BF, Shahda S, O’Neil BH, Noonan AM, et al. Phase 1b/2 trial of cancer stemness inhibitor napabucasin (NAPA) + nab-paclitaxel (nPTX) and gemcitabine (Gem) in metastatic pancreatic adenocarcinoma (mPDAC). Journal of Clinical Oncology. 2018;36(15_suppl):4110-.

20. Hubbard JM, Grothey A. Napabucasin: An Update on the First-in-Class Cancer Stemness Inhibitor. Drugs. 2017;77(10):1091-103. [PubMed: 28573435]

21. Li Y, Rogoff HA, Keates S, Gao Y, Murikipudi S, Mikule K, et al. Suppression of cancer relapse and metastasis by inhibiting cancer stemness. Proc Natl Acad Sci U S A. 2015;112(6):1839-44. [PubMed: 25605917]

22. Zuo D, Shogren KL, Zang J, Jewison DE, Waletzki BE, Miller AL 2nd, et al. Inhibition of STAT3 blocks protein synthesis and tumor metastasis in osteosarcoma cells. Journal of experimental \& clinical cancer research : CR. 2018;37(1):244. [PubMed: 30286779]

23. Li C, Chen C, An Q, Yang T, Sang Z, Yang Y, et al. A novel series of napabucasin derivatives as orally active inhibitors of signal transducer and activator of transcription 3 (STAT3). European journal of medicinal chemistry. 2018;162:543-54. [PubMed: 30472602]

24. Boj SF, Hwang CI, Baker LA, Chio II, Engle DD, Corbo V, et al. Organoid models of human and mouse ductal pancreatic cancer. Cell. 2015;160(1-2):324-38. [PubMed: 25557080]

25. Shi J, Wang E, Milazzo JP, Wang Z, Kinney JB, Vakoc CR. Discovery of cancer drug targets by CRISPR-Cas9 screening of protein domains. Nat Biotechnol. 2015;33(6):661-7. [PubMed: 25961408]

26. Roe JS, Hwang CI, Somerville TDD, Milazzo JP, Lee EJ, Da Silva B, et al. Enhancer Reprogramming Promotes Pancreatic Cancer Metastasis. Cell. 2017;170(5):875-88.e20. [PubMed: 28757253]

27. Moore T, Le A, Niemi AK, Kwan T, Cusmano-Ozog K, Enns GM, et al. A new LC-MS/MS method for the clinical determination of reduced and oxidized glutathione from whole blood. Journal of chromatography B, Analytical technologies in the biomedical and life sciences. 2013;929:51-5. [PubMed: 23660247] 
28. Love MI, Huber W, Anders S. Moderated estimation of fold change and dispersion for RNA-seq data with DESeq2. Genome biology. 2014;15(12):550. [PubMed: 25516281]

29. Subramanian A, Tamayo P, Mootha VK, Mukherjee S, Ebert BL, Gillette MA, et al. Gene set enrichment analysis: a knowledge-based approach for interpreting genome-wide expression profiles. Proc Natl Acad Sci U S A. 2005;102(43):15545-50. [PubMed: 16199517]

30. Szklarczyk D, Franceschini A, Kuhn M, Simonovic M, Roth A, Minguez P, et al. The STRING database in 2011: functional interaction networks of proteins, globally integrated and scored. Nucleic Acids Res. 2011;39(Database issue):D561-8. [PubMed: 21045058]

31. Doncheva NT, Morris JH, Gorodkin J, Jensen LJ. Cytoscape StringApp: Network Analysis and Visualization of Proteomics Data. Journal of proteome research. 2019;18(2):623-32. [PubMed: 30450911]

32. Shannon P, Markiel A, Ozier O, Baliga NS, Wang JT, Ramage D, et al. Cytoscape: a software environment for integrated models of biomolecular interaction networks. Genome Res. 2003;13(11):2498-504. [PubMed: 14597658]

33. Huang X, Motea EA, Moore ZR, Yao J, Dong Y, Chakrabarti G, et al. Leveraging an NQO1 Bioactivatable Drug for Tumor-Selective Use of Poly(ADP-ribose) Polymerase Inhibitors. Cancer Cell. 2016;30(6):940-52. [PubMed: 27960087]

34. Alam J, Stewart D, Touchard C, Boinapally S, Choi AM, Cook JL. Nrf2, a Cap'n'Collar transcription factor, regulates induction of the heme oxygenase-1 gene. J Biol Chem. 1999;274(37):26071-8. [PubMed: 10473555]

35. Dunn LL, Midwinter RG, Ni J, Hamid HA, Parish CR, Stocker R. New insights into intracellular locations and functions of heme oxygenase-1. Antioxidants \& redox signaling. 2014;20(11):172342. [PubMed: 24180287]

36. Reczek CR, Birsoy K, Kong H, Martinez-Reyes I, Wang T, Gao P, et al. A CRISPR screen identifies a pathway required for paraquat-induced cell death. Nature chemical biology. 2017;13(12):1274-9. [PubMed: 29058724]

37. Chio IIC, Tuveson DA. ROS in Cancer: The Burning Question. Trends Mol Med. 2017;23(5):41129. [PubMed: 28427863]

38. Trachootham D, Alexandre J, Huang P. Targeting cancer cells by ROS-mediated mechanisms: a radical therapeutic approach? Nat Rev Drug Discov. 2009;8(7):579-91. [PubMed: 19478820]

39. Chiarugi P PTPs versus PTKs: the redox side of the coin. Free radical research. 2005;39(4):35364. [PubMed: 16028361]

40. Kasiappan R, Jutooru I, Karki K, Hedrick E, Safe S. Benzyl Isothiocyanate (BITC) Induces Reactive Oxygen Species-dependent Repression of STAT3 Protein by Down-regulation of Specificity Proteins in Pancreatic Cancer. J Biol Chem. 2016;291(53):27122-33. [PubMed: 27875298]

41. Sobotta MC, Liou W, Stocker S, Talwar D, Oehler M, Ruppert T, et al. Peroxiredoxin-2 and STAT3 form a redox relay for $\mathrm{H} 2 \mathrm{O} 2$ signaling. Nature chemical biology. 2015;11(1):64-70. [PubMed: 25402766]

42. Jonker DJ, Nott L, Yoshino T, Gill S, Shapiro J, Ohtsu A, et al. Napabucasin versus placebo in refractory advanced colorectal cancer: a randomised phase 3 trial. The lancet Gastroenterology \& hepatology. 2018;3(4):263-70. [PubMed: 29397354]

43. Gorrini C, Harris IS, Mak TW. Modulation of oxidative stress as an anticancer strategy. Nat Rev Drug Discov. 2013;12(12):931-47. [PubMed: 24287781]

44. Bey EA, Reinicke KE, Srougi MC, Varnes M, Anderson VE, Pink JJ, et al. Catalase abrogates beta-lapachone-induced PARP1 hyperactivation-directed programmed necrosis in NQO1-positive breast cancers. Mol Cancer Ther. 2013;12(10):2110-20. [PubMed: 23883585]

45. Harris IS, Treloar AE, Inoue S, Sasaki M, Gorrini C, Lee KC, et al. Glutathione and thioredoxin antioxidant pathways synergize to drive cancer initiation and progression. Cancer Cell. 2015;27(2):211-22. [PubMed: 25620030]

46. Gout PW, Buckley AR, Simms CR, Bruchovsky N. Sulfasalazine, a potent suppressor of lymphoma growth by inhibition of the $\mathrm{x}(\mathrm{c})$ - cystine transporter: a new action for an old drug. Leukemia. 2001;15(10):1633-40. [PubMed: 11587223] 
47. Marzano C, Gandin V, Folda A, Scutari G, Bindoli A, Rigobello MP. Inhibition of thioredoxin reductase by auranofin induces apoptosis in cisplatin-resistant human ovarian cancer cells. Free radical biology \& medicine. 2007;42(6):872-81. [PubMed: 17320769] 


\section{Statement of translational relevance}

Napabucasin is an orally administered small molecule currently undergoing clinical evaluation for treatment of cancer. It has been proposed to exert its anti-cancer activity by inhibiting STAT3 signaling and cancer stemness properties. Here, we show that napabucasin is a quinone that is bioactivated by oxidoreductases, in particular $\mathrm{NAD}(\mathrm{P}) \mathrm{H}$ :quinone oxidoreductase 1 (NQO1) and to a lesser extent the Cytochrome P450 oxidoreductase (POR). Bioactivation of napabucasin generates cytotoxic levels of reactive oxygen species (ROS) resulting in DNA damage-induced cell death and multiple ROS-induced intracellular events, including a reduction in STAT3 phosphorylation. This better understanding of the mechanism of action of napabucasin will assist the development of novel, more effective therapeutic combination approaches, and will also aid in the identification of potential biomarkers of patients likely to respond to napabucasin. 
A

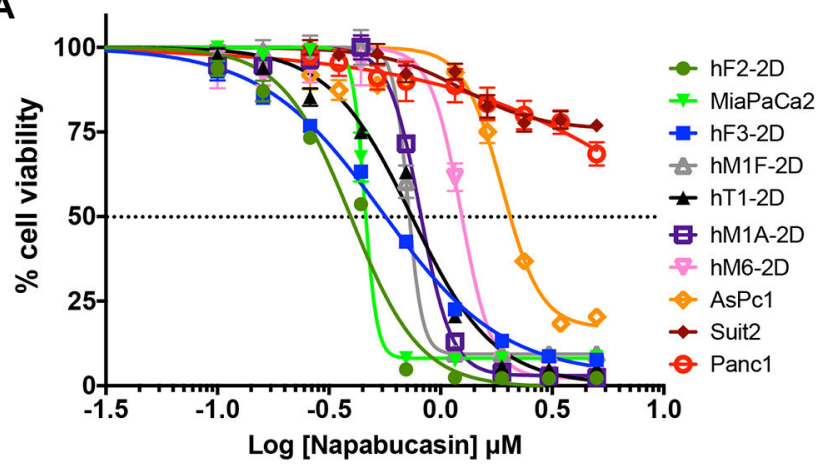

B

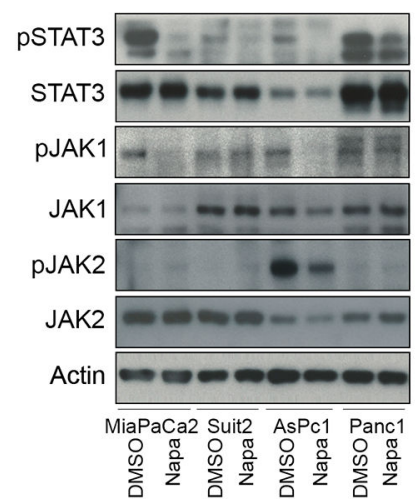

C - DMSO - Napabucasin
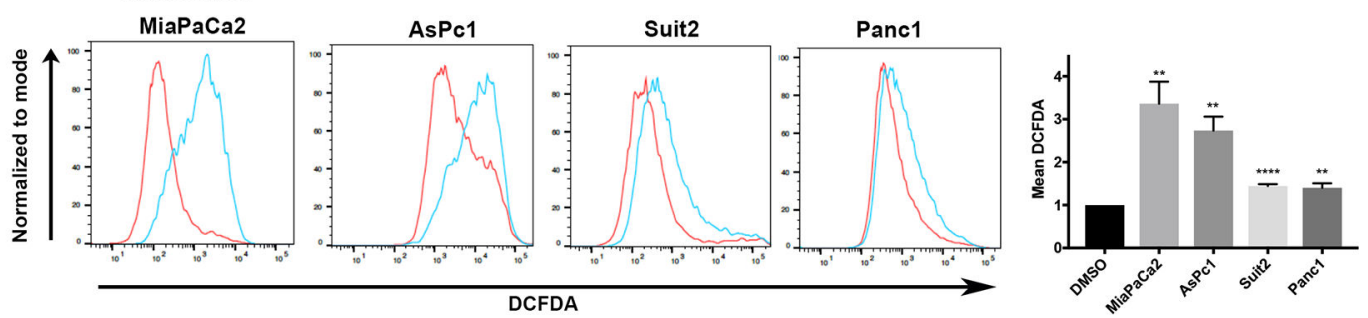

D

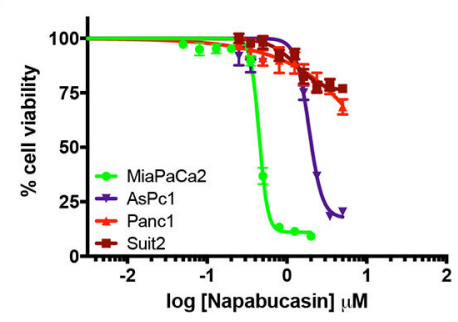

$\mathbf{F}$

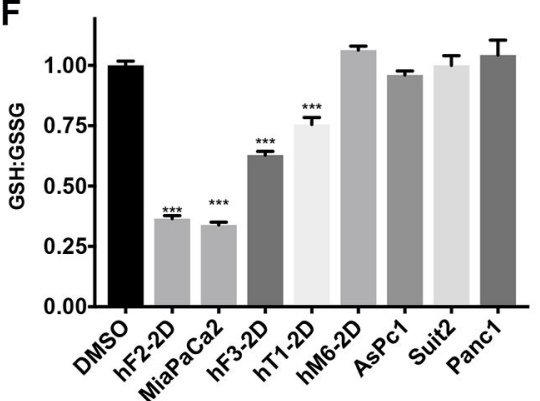

E

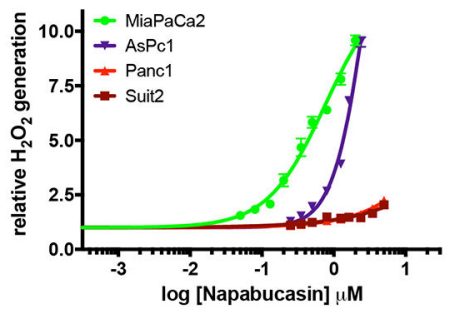

G

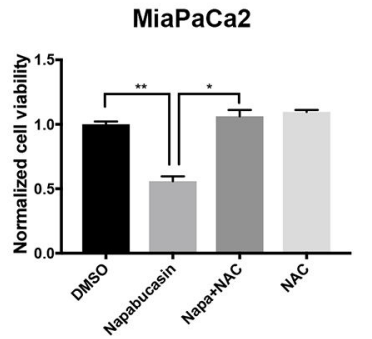

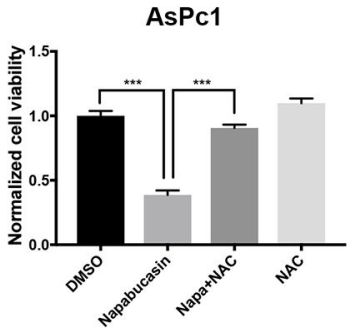

Figure 1. Treatment with napabucasin induces ROS

(A) Cell viability of a variety of pancreatic cancer cell lines treated with increasing concentrations of napabucasin for 6 hours. Results show mean \pm SEM of 3-5 biological replicates. (B) Western blot analysis of pSTAT3, STAT3, pJAK1, JAK1, pJAK2 and JAK2 expression after 2 hours of treatment with DMSO as vehicle control or $0.5 \mu \mathrm{M}$ napabucasin for MiaPaCa2 cells, 1.0 $\mu \mathrm{M}$ napabucasin for Suit2, and Panc1 cells and 2.0 $\mu \mathrm{M}$ for AsPc1 cells, with Actin as loading control. (C) ROS generation following 6 hours of treatment with napabucasin measured by $\mathrm{CM}$ (chloromethyl)- $\mathrm{H}_{2}$ DCFDA staining. Representative images of 
3 biological replicates, with quantification of the mean DCFDA staining, are shown for indicated cell lines treated with napabucasin at concentrations as in (B). (D) Cell viability and (E) $\mathrm{H}_{2} \mathrm{O}_{2}$ generation in pancreatic cancer cells treated with increasing concentrations of napabucasin for 6 hours. Results show mean \pm SEM of 3 biological replicates. (F) Ratio of glutathione (GSH) to glutathione disulfide (GSSG) in indicated cell lines treated for 6 hours with $0.5 \mu \mathrm{M}$ napabucasin. Results show mean \pm SEM of 4 biological replicates. (G) cell viability of MiaPaCa2 and AsPc1 cells treated for 6 hours with napabucasin as single agent or combined with $1.25 \mathrm{mM}$ NAC. Results show mean \pm SEM of 3 biological replicates. Unpaired two-tailed t-test $* \mathrm{p}<0.05, * * \mathrm{p}<0.01, * * * \mathrm{p}<0.001, * * * * \mathrm{p}<0.0001$ 

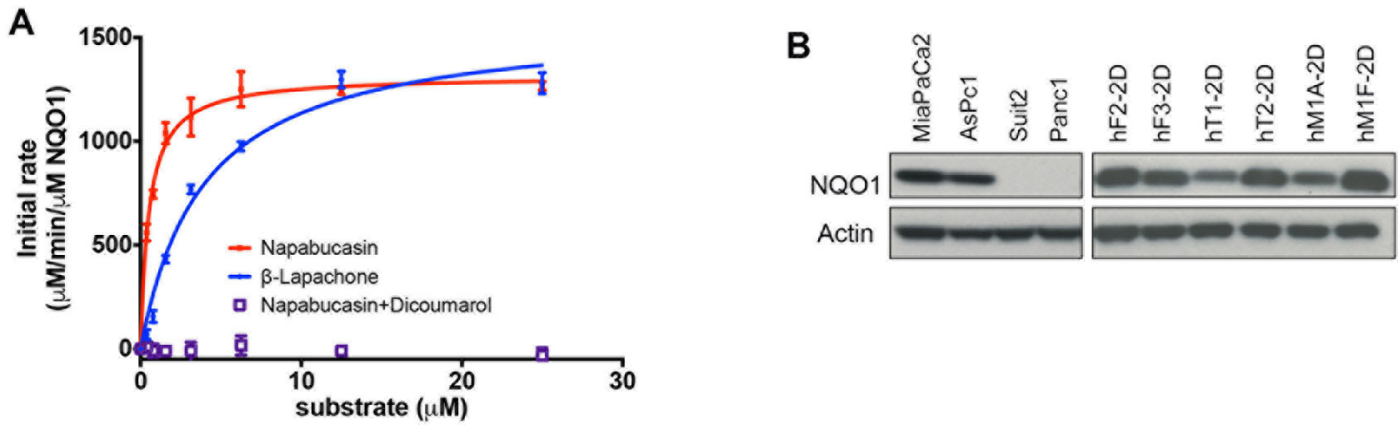

\begin{tabular}{|l|c|c|}
\hline & Napabucasin & $\beta$-Lapachone \\
\hline $\mathbf{K}_{\mathbf{M}}(\mu \mathrm{M})$ & $0.5(0.4-0.6)$ & $3.9(3.4-4.4)$ \\
$\boldsymbol{K}_{\text {cat }}\left(\mathbf{m i n}^{-1}\right)$ & $1316(1286-1346)$ & $1580(1514-1646)$ \\
$\mathbf{K}_{\text {ca: }} / \mathbf{K}_{\mathbf{M}}\left(\mathbf{M}^{-1} \mathbf{s e c}^{-1}\right)$ & $4.4 \mathrm{E}+07$ & $6.8 \mathrm{E}+06$ \\
\hline
\end{tabular}

C

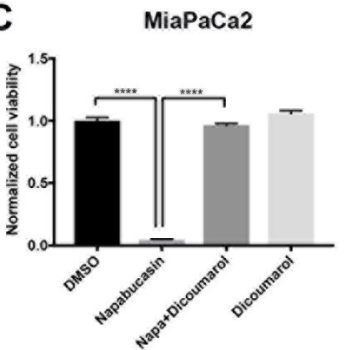

D

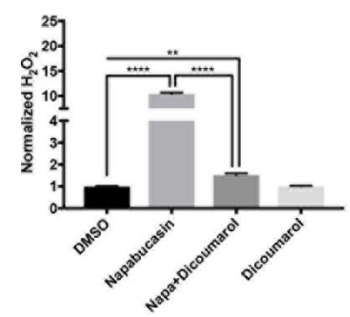

E

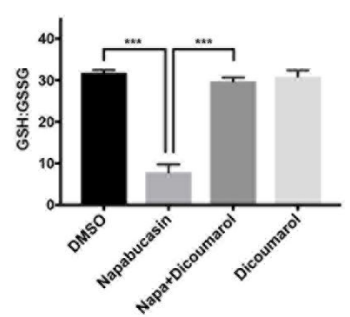

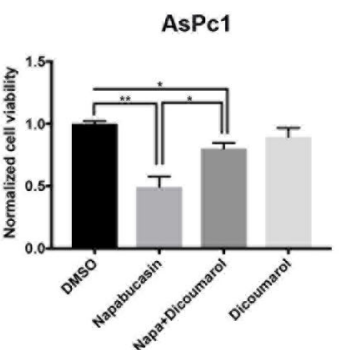

AsPc1

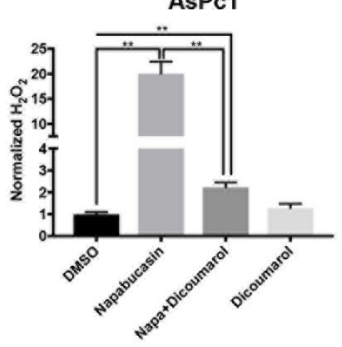

AsPc1

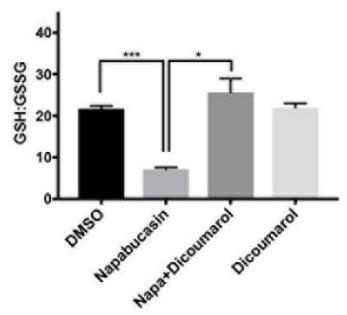

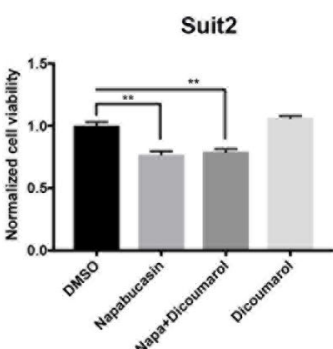

Suit2

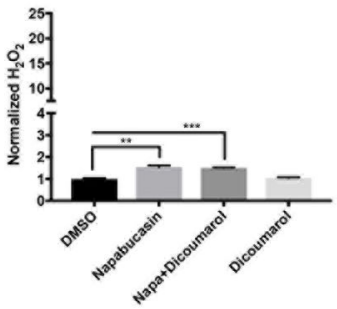

Suit2

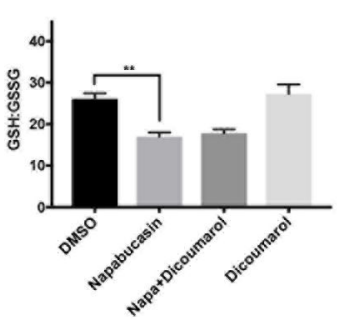

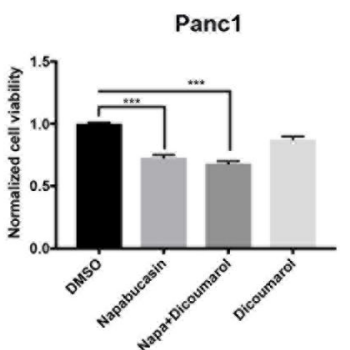

Panc1
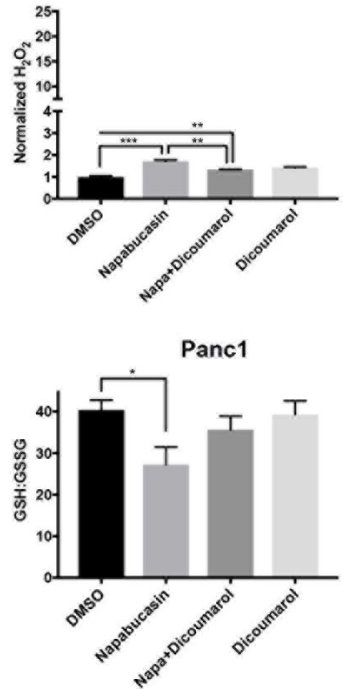

Figure 2. Napabucasin is an NQO1 substrate

(A) Cell-free assay measuring depletion of NADPH in the presence of NQO1 plus increasing concentration of napabucasin as single agent or combined with $5 \mathrm{mM}$ dicoumarol or NQO1 plus increasing concentration of $\beta$-lapachone. Results show mean \pm SEM of 3 biological replicates, with quantification showing the mean calculated affinity $\left(\mathrm{K}_{\mathrm{M}}\right)$, rate $\left(\mathrm{K}_{\text {cat }}\right)$, and enzymatic efficiency $\left(\mathrm{K}_{\text {cat }} / \mathrm{K}_{\mathrm{M}}\right)$ with $95 \%$ confidence intervals. (B) Western blot analysis of NQO1 expression in a panel of pancreatic cancer cell lines, with Actin as loading control. (C) Cell viability and (D) $\mathrm{H}_{2} \mathrm{O}_{2}$ generation after 6 hours of treatment with DMSO as 
vehicle control or with napabucasin as single agent or combined with the NQO1 inhibitor dicoumarol at $10 \mu \mathrm{M}$. Dicoumarol treatment as single agent is shown as control. (E) Ratio of glutathione (GSH) to glutathione disulfide (GSSG) in napabucasin-treated cell lines cultured in the absence and presence of $10 \mu \mathrm{M}$ dicoumarol for 24 hours. Napabucasin concentrations used were $0.5 \mu \mathrm{M}$ for MiaPaCa2, $1.0 \mu \mathrm{M}$ for Panc1 and Suit2 and $2.0 \mu \mathrm{M}$ for AsPc1. Results show mean \pm SEM of 3 biological replicates, unpaired two-tailed t-test $* \mathrm{p}<0.05$, $* * \mathrm{p}<0.01$, $* * * \mathrm{p}<0.001, * * * * \mathrm{p}<0.0001$. 
A

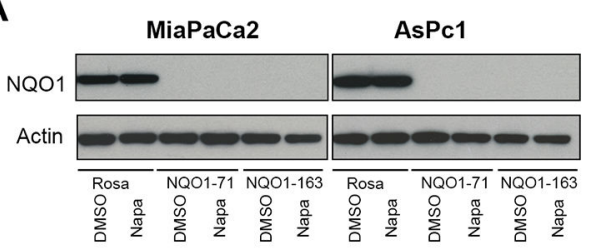

B
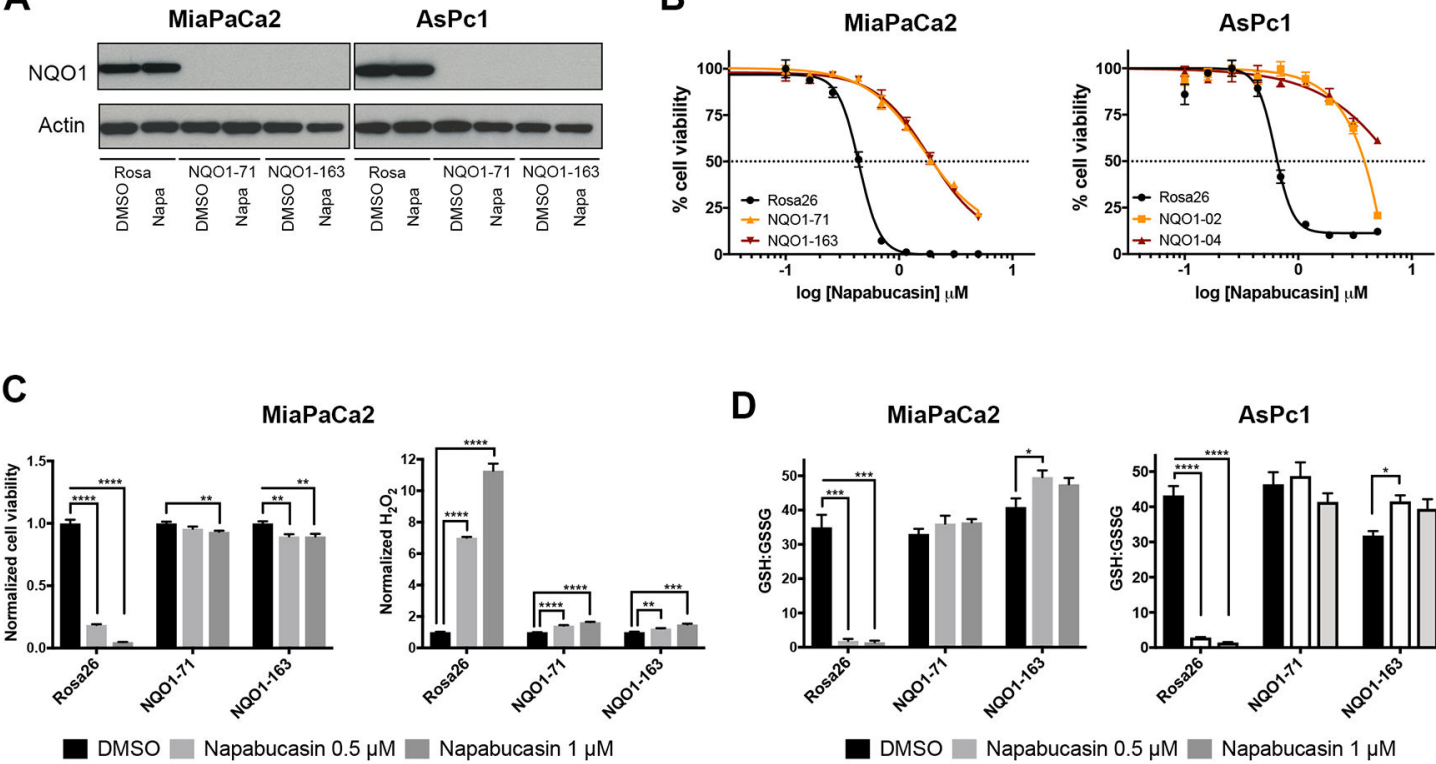

D

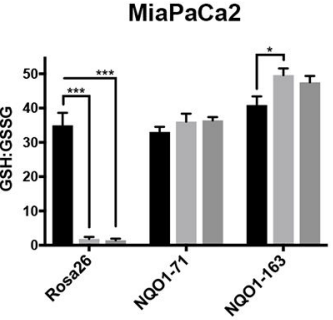

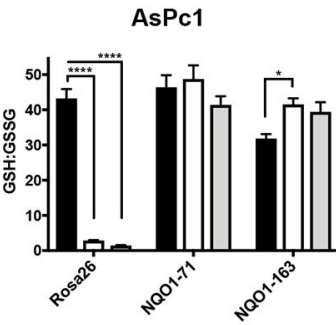

DMSO Napabucasin $0.5 \mu \mathrm{M} \quad$ Napabucasin $1 \mu \mathrm{M}$ $\square$ Napabucasin $2 \mu \mathrm{M} \quad \square$ Napabucasin $3 \mu \mathrm{M}$
E

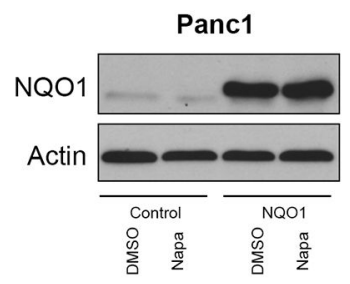

$\mathbf{F}$

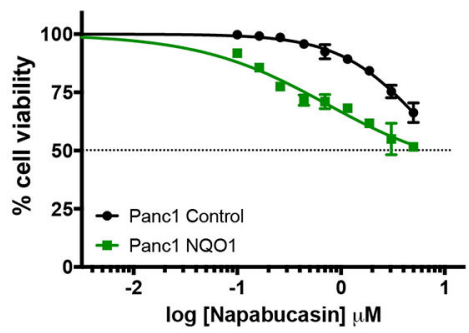

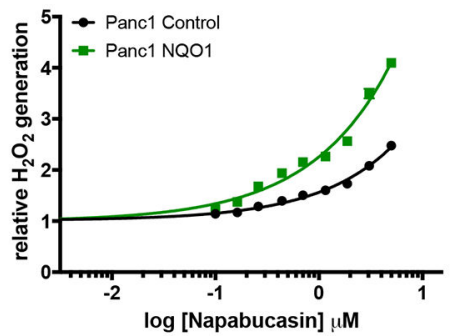

G

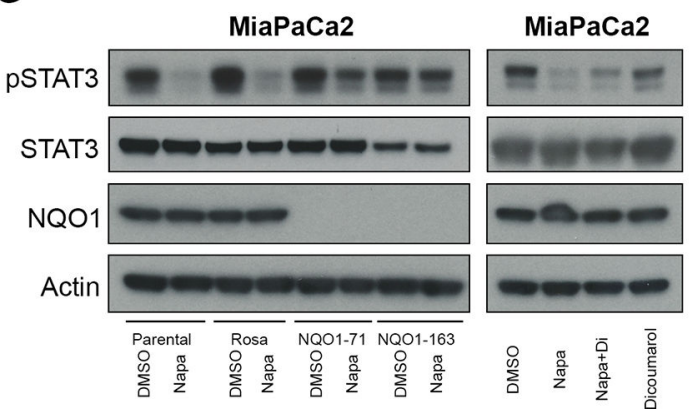

H

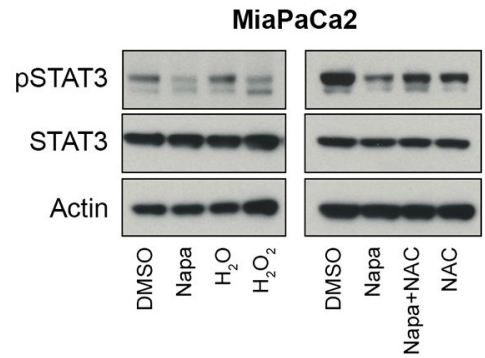

Figure 3. Activity of napabucasin requires NQO1

(A) Western blot analysis for indicated CRISPR clones confirming knock-out of NQO1 expression in $\mathrm{MiaPaCa} 2$ and $\mathrm{AsPc} 1$ cells with no changes in expression upon 2-hour treatment with napabucasin. Actin is shown as loading control. (B) Cell viability of $\mathrm{MiaPaCa} 2$ and AsPc1 NQO1 CRISPR clones treated with increasing concentrations of napabucasin for 6 hours. (C) Cell viability and $\mathrm{H}_{2} \mathrm{O}_{2}$ generation in MiaPaCa2 NQO1 CRISPR clones following 6 hours of napabucasin treatment at the indicated concentrations. (D) Ratio of glutathione (GSH) to glutathione disulfide (GSSG) in MiaPaCa2 and AsPc1 
NQO1 CRISPR clones after 24 hours of napabucasin treatment at the indicated concentrations. Results show mean \pm SEM of 3 biological replicates, unpaired two-tailed ttest $* \mathrm{p}<0.05, * * \mathrm{p}<0.01, * * * \mathrm{p}<0.001, * * * * \mathrm{p}<0.0001$. (E) Western blot analysis of NQO1 expression in Panc1 cells expressing a CMV-GFP control plasmid and Panc1 cells with ectopic NQO1 expression, treated for 2 hours with $1.0 \mu \mathrm{M}$ napabucasin. Actin is shown as loading control. (F) Cell viability and $\mathrm{H}_{2} \mathrm{O}_{2}$ generation in Panc1 clones as in (E) treated with increasing concentrations of napabucasin for 6 hours. (G) Western blot analysis of pSTAT3, STAT3 and NQO1 expression in indicated MiaPaCa2 cells or CRISPR clones treated for 2 hours with $0.5 \mu \mathrm{M}$ napabucasin as single agent or combined with $10 \mu \mathrm{M}$ dicoumarol, with actin as loading control $(\mathrm{H})$ Western blot analysis of pSTAT3 and STAT3 expression in MiaPaCa2 cells treated for 2 hours with $0.5 \mu \mathrm{M}$ napabucasin, with $200 \mu \mathrm{M} \mathrm{H}_{2} \mathrm{O}_{2}$, napabucasin combined with $1.25 \mathrm{mM} \mathrm{NAC}$ or NAC as single agent. DMSO and $\mathrm{H}_{2} \mathrm{O}$ are used as respective vehicle controls. Actin is shown as loading control. 
A

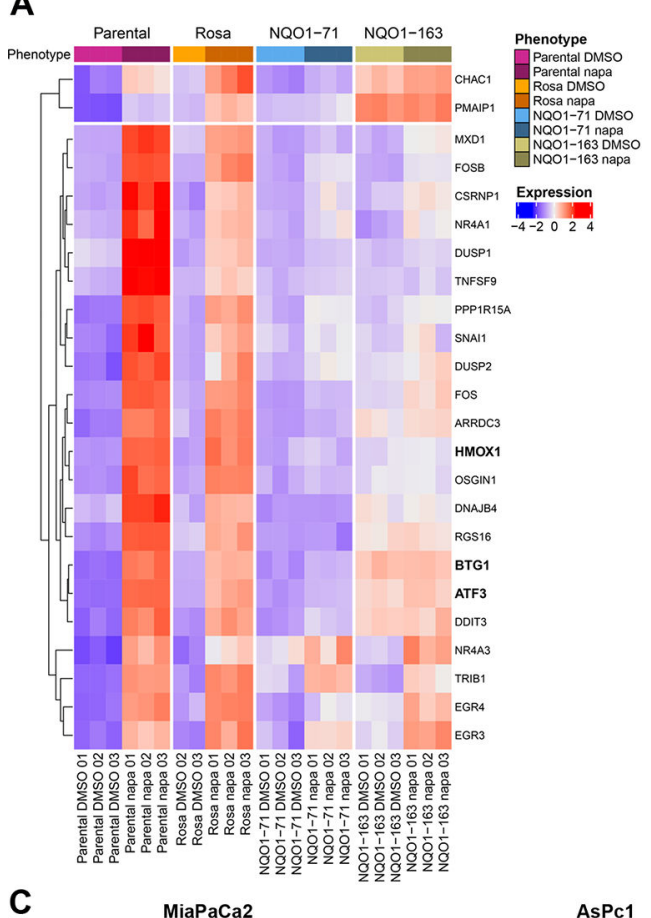

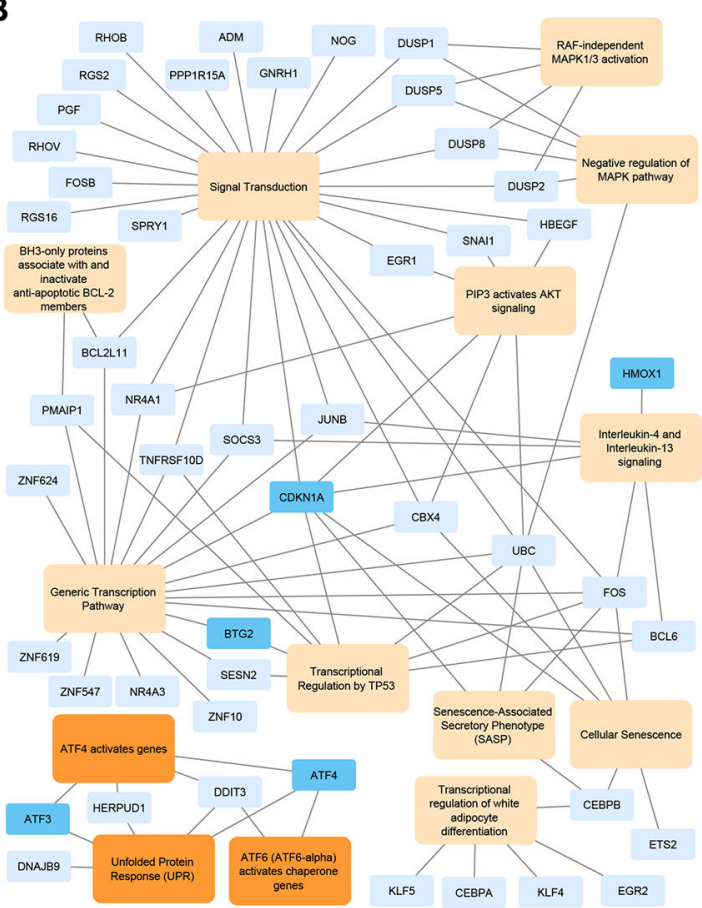

D
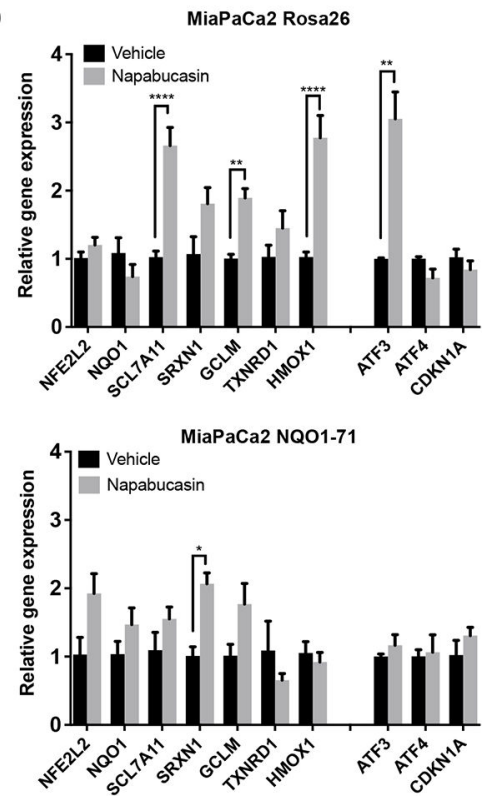

Figure 4. Napabucasin induces ROS and cellular stress

(A) Heatmap showing genes that are significantly differentially expressed (adjusted p-value $<0.05, \log 2$ fold change $>=1$ ) in both parental and Rosa26 control MiaPaCa2 cells but not in the NQO1-71 and NQO1-163 MiaPaCa2 CRISPR clones following 2-hour treatment with $0.5 \mu \mathrm{M}$ napabucasin or DMSO as vehicle control. (B) Network of pathway-protein interactions from significantly enriched Reactome pathways in parental MiaPaCa2 cells. (C) qPCR analysis of NFE2L2/NRF2 and a selection of NRF2 target genes in the indicated cell lines treated for 2 hours with DMSO as vehicle control or $0.5 \mu \mathrm{M}$ napabucasin for 
MiaPaCa2 cells, $1.0 \mu \mathrm{M}$ napabucasin for Suit2, and Panc1 cells and $2.0 \mu \mathrm{M}$ for AsPc1 cells. (D) qPCR analysis of snap-frozen tumor samples from MiaPaCa2 Rosa26 xenografts ( $\mathrm{n}=6$ ) and MiaPaCa2 NQO1-71 xenografts $(\mathrm{n}=6)$ treated for 24 days with napabucasin or vehicle control for expression of indicated genes. (E) qPCR analysis of ATF3, ATF4 and CKN1A expression in the indicated cell lines treated for 2 hours as in (C). Results in (C), (D) and (E) show mean \pm SEM of 3 biological replicates, unpaired two-tailed t-test $* \mathrm{p}<0.05$, ${ }^{* *} \mathrm{p}<0.01$, $* * * \mathrm{p}<0.001, * * * * \mathrm{p}<0.0001$. 
A

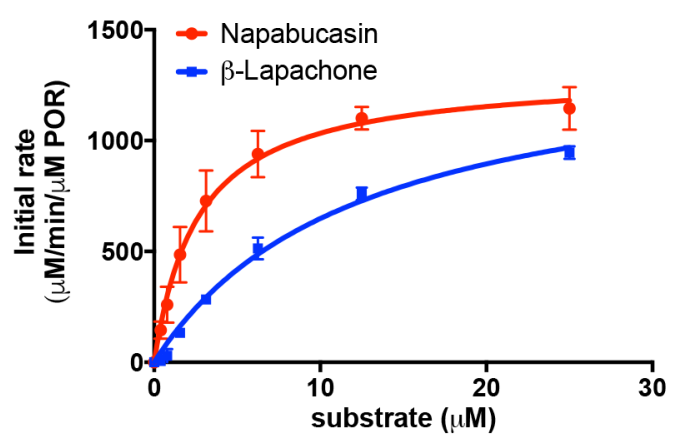

C

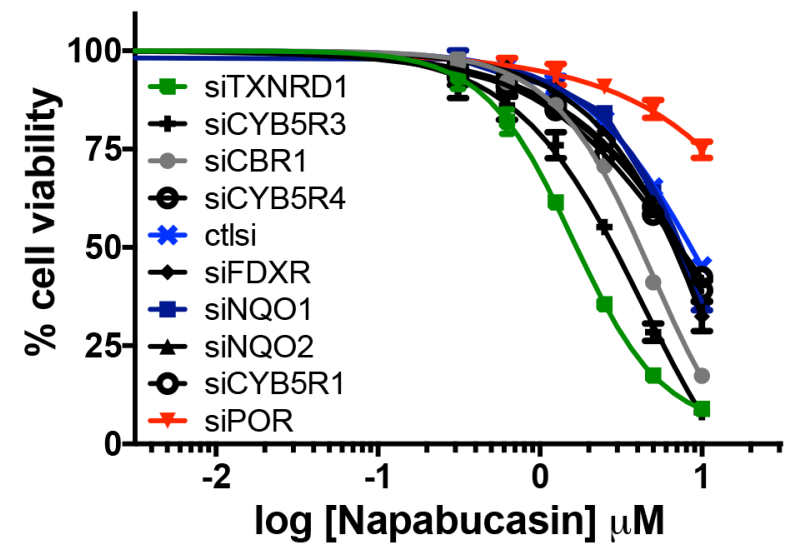

\begin{tabular}{|l|c|c|}
\hline & Napabucasin & $\beta$-Lapachone \\
\hline $\mathbf{K}_{\mathbf{M}}(\boldsymbol{\mu M})$ & $2.6(2.0-3.2)$ & $11.3(9.5-13.1)$ \\
$\mathbf{K}_{\text {cat }}\left(\mathbf{m i n}^{-1}\right)$ & $1306(1220-1392)$ & $1444(1344-1544)$ \\
$\mathbf{k}_{\text {cat }} / \mathbf{K}_{\mathbf{M}}\left(\mathbf{M}^{-1} \mathbf{s e c}^{-1}\right)$ & $8.3 E+06$ & $2.1 E+06$ \\
\hline
\end{tabular}

B

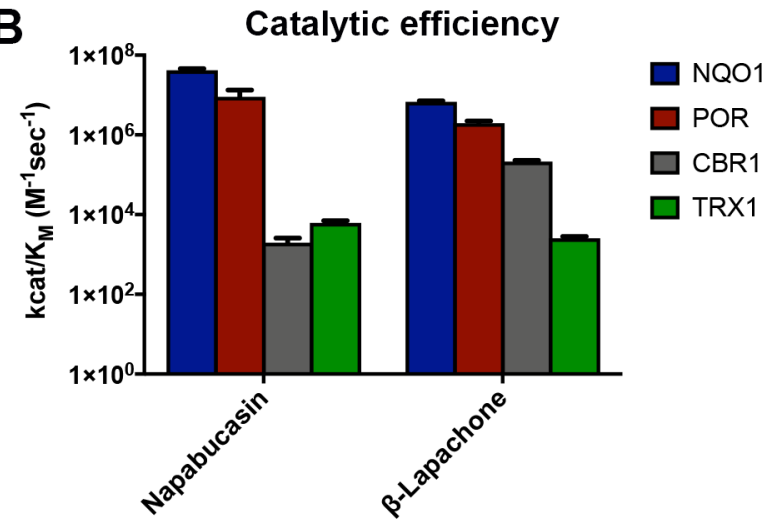

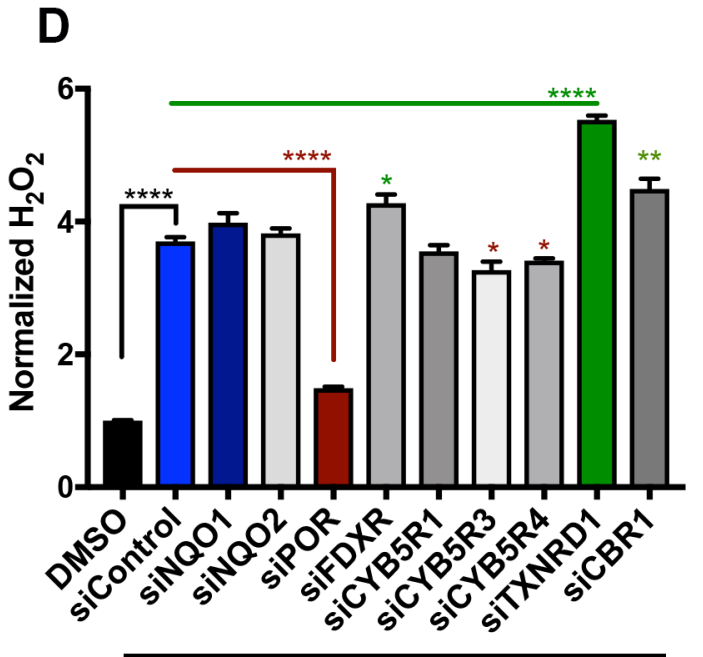

Napabucasin

Figure 5. Role of additional cellular reductases in the antitumor activity of napabucasin (A) Cell-free assay measuring depletion of NADPH in the presence of POR plus napabucasin or $\beta$-lapachone with quantification of the reactions performed showing the mean calculated affinity $\left(\mathrm{K}_{\mathrm{M}}\right)$, rate $\left(\mathrm{K}_{\mathrm{cat}}\right)$, and enzymatic efficiency $\left(\mathrm{K}_{\mathrm{cat}} / \mathrm{K}_{\mathrm{M}}\right)$ with $95 \%$ confidence intervals. (B) Cell-free assay measuring depletion of NADPH in the presence of NQO1, POR, CBR1, or TRX1 and either napabucasin or $\beta$-lapachone. (C) Cell viability of Panc1 cells with siRNA-mediated knock-down of a variety of cellular reductases upon treatment for 6 hours with increasing concentrations of napabucasin. (D) $\mathrm{H}_{2} \mathrm{O}_{2}$ generation in Panc1 cells with siRNA-mediated knock-down of the indicated reductases after 6 hours of treatment with $5 \mu \mathrm{M}$ napabucasin (or DMSO as vehicle control). Results show mean \pm SEM of 3 biological replicates, unpaired two-tailed t-test $* \mathrm{p}<0.05, * * \mathrm{p}<0.01, * * * * \mathrm{p}<0.0001$. 
A

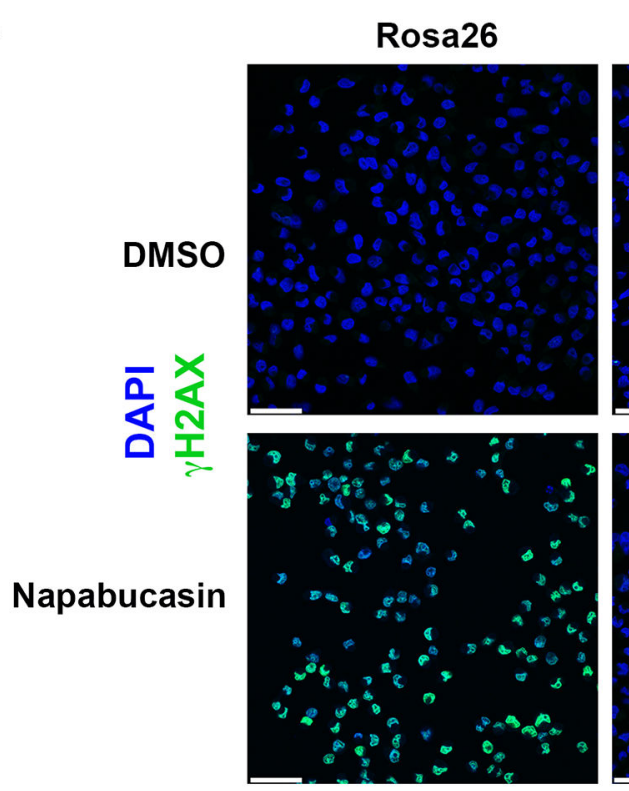

B

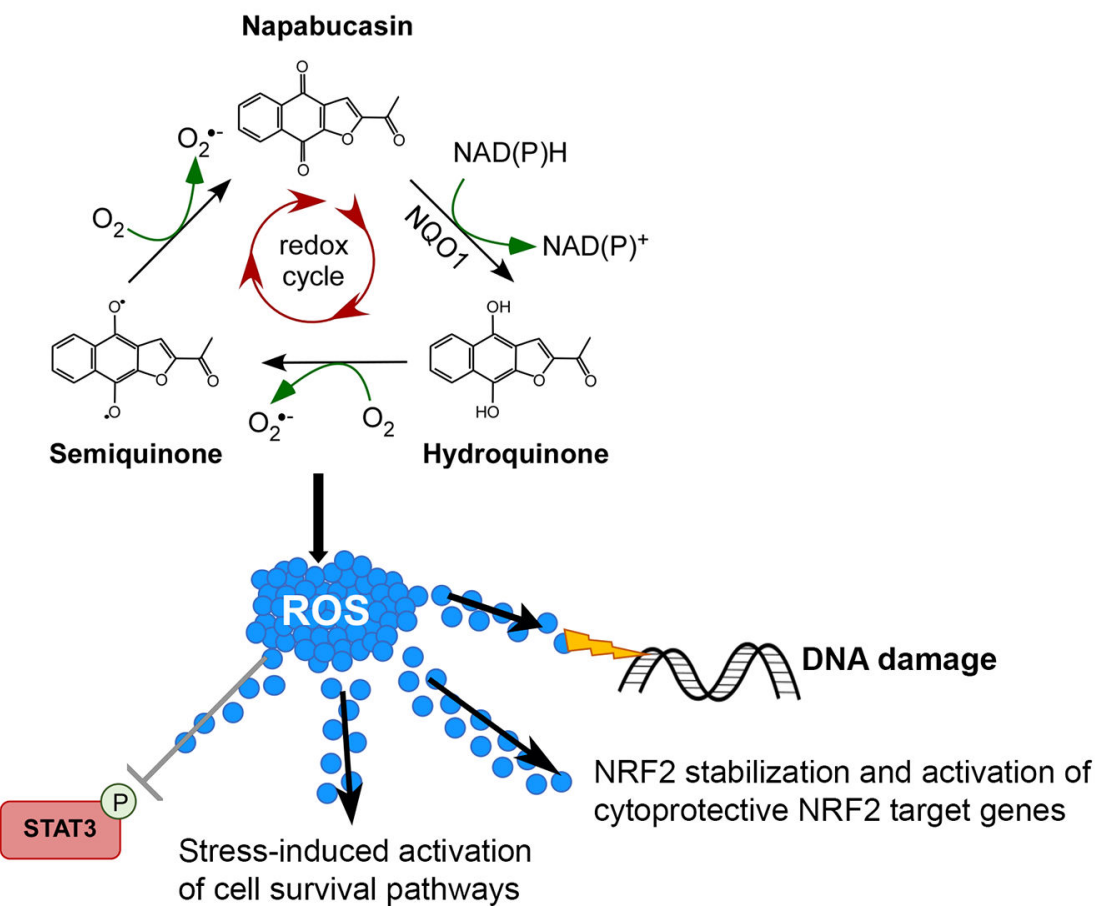

Figure 6. Proposed mechanism of action of napabucasin

(A) Representative images of immunofluorescence showing marked induction of $\gamma \mathrm{H} 2 \mathrm{AX}$ (green) in MiaPaCa2 Rosa26 cells but not in MiaPaCa2 NQO1-71 and NQO-163 CRISPR clones treated for 6 hours with $0.5 \mu \mathrm{M}$ napabucasin or DMSO as vehicle control. DAPI (blue) was used as counter stain. Scale bar $50 \mu \mathrm{m}$. (B) Cartoon of the proposed mechanism of action of the naphthoquinone napabucasin. 